\title{
Generalized traveling-wave method, variational approach, and modified conserved quantities for the perturbed nonlinear Schrödinger equation
}

\author{
Niurka R. Quintero* \\ Departamento de Física Aplicada I, E.U.P., Universidad de Sevilla, c/Virgen de África 7, 41011 Sevilla, Spain \\ Franz G. Mertens ${ }^{\dagger}$ \\ Physikalisches Institut, Universität Bayreuth, D-95440 Bayreuth, Germany
}

A. R. Bishop
Theoretical Division and Center for Nonlinear Studies, Los Alamos National Laboratory, Los Alamos, New Mexico 87545, USA

(Received 16 March 2010; revised manuscript received 8 June 2010; published 29 July 2010)

\begin{abstract}
The generalized traveling wave method (GTWM) is developed for the nonlinear Schrödinger equation (NLSE) with general perturbations in order to obtain the equations of motion for an arbitrary number of collective coordinates. Regardless of the particular ansatz that is used, it is shown that this alternative approach is equivalent to the Lagrangian formalism, but has the advantage that only the Hamiltonian of the unperturbed system is required, instead of the Lagrangian for the perturbed system. As an explicit example, we take 4 collective coordinates, namely the position, velocity, amplitude and phase of the soliton, and show that the GTWM yields the same equations of motion as the perturbation theory based on the Inverse Scattering Transform and as the time variation of the norm, first moment of the norm, momentum, and energy for the perturbed NLSE.
\end{abstract}

DOI: 10.1103/PhysRevE.82.016606

PACS number(s): 05.45.Yv

\section{INTRODUCTION}

With great generality, and in many phenomena, such as scattering and diffusion, solitons, and other nonlinear coherent excitations in extended systems behave like particles $[1,2]$. This fact allows one to reduce the infinite number of degrees of freedom of the extended systems to only a few, when we are interested in the behavior of the soliton excitations. E.g., the dynamics of topological solitons of the nonlinear Klein-Gordon equations (NLKGEs) in the simplest case can be described in terms of one so-called collective coordinate $(\mathrm{CC})[3,4]$, typically the center of the soliton. More sophisticated Ansätze take into account other degrees of freedom, using two CCs [5,6], three CCs [7], or even more [8].

The dynamics of nontopological solitons of the nonlinear Schrödinger equation (NLSE) is more complicated: The bright one-soliton solution, which has an internal oscillation, depends on four parameters [2]. Therefore, typically four $\mathrm{CCs}$, namely position, amplitude, velocity and phase, have been used.

There are several methods to determine the equations of motion that the CCs satisfy. For instance, for modified NLSEs several perturbation theories can be applied [9-12], for certain systems one can use the perturbed inverse scattering transform (IST) $[13,14]$, and if a Lagrangian density exists one can derive Lagrange equations, which are the evolution equations of the CCs [15-17]. Moreover, the time variation of $M$ conserved quantities of the unperturbed sys-

\footnotetext{
*niurka@us.es

†ranz.mertens@uni-bayreuth.de
}

tem generally is related with the evolution of $M$ CCs $[18,19]$.

There is another method, the so-called generalized traveling wave method (GTWM), in which a certain projection technique is used to obtain the equations of motion of the CCs. The GTWM was introduced in a general way in Ref. [20]: only the Hamilton equations of the unperturbed system must be known and the unperturbed system need not be integrable. The method was applied to the zero-temperature dynamics [20] and the thermal diffusion $[21,22]$ of magnetic vortices in the two-dimensional (2D) anisotropic Heisenberg model. The GTWM was also applied to the dynamics of topological solitons in NLKGEs [23]. Together with the Rice ansatz $[5,6]$, which introduces the position and the width of the soliton as CCs, the method explained resonances due to the action of an ac force [23-25] and described transport phenomena of ratchet type in sine-Gordon and $\phi^{4}$ models with biharmonic driving and damping [26,27]. The sineGordon model was applied, e.g., to long Josephson junctions, where the fluxons play the role of the solitons, and the ratchet effect was confirmed by experiments [28].

The aim of this work is to develop the GTWM for an arbitrary ansatz with $M$ CCs for the perturbed NLSE, see Sec. II. Furthermore, we show in Sec. III that in cases where the Lagrangian density for the perturbed system exists, the GTWM is equivalent to the variational approach. In particular, by using only 4 CCs we show in Sec. IV that the GTWM is also equivalent to the results obtained from the perturbation theory based on the inverse scattering transform [14], from the adiabatic perturbation theory [29] and from the variation of the norm, the first moment of the norm, the momentum, and the energy of the perturbed NLSE. Finally in Sec. V, we summarize our main results. 


\section{GENERALIZED TRAVELING WAVE METHOD}

The perturbed NLSE actually consists of two equations for the real and imaginary parts, or for $u(x, t)$ and $u^{*}(x, t)$. Namely,

$$
i u_{t}+u_{x x}+2|u|^{2} u=R[u(x, t) ; x, t],
$$

and its complex conjugate equation, where $R[u(x, t) ; x, t]$ represents a general perturbation, which may also depend on $u^{*}$ and the spatial derivatives of $u$ and $u^{*}$. This system has very many applications in practically all fields of physics, which are listed and discussed in several reviews articles $[14-16,30]$. In the case of optical solitons, $t$ and $x$ are replaced by the propagation distance and the pulse duration, respectively.

For our purpose, we rewrite Eq. (1)

$$
i u_{t}=\frac{\delta H_{0}}{\delta u^{*}}+R[u(x, t) ; x, t],
$$

where

$$
H_{0}=\int_{-\infty}^{+\infty} d x \mathcal{H}_{0}=\int_{-\infty}^{+\infty} d x\left(u_{x} u_{x}^{*}-u^{2} u^{* 2}\right) .
$$

For the following, only this Hamiltonian of the unperturbed system must be known. We now assume that the time dependence of $u(x, t)$ and $u^{*}(x, t)$ in Eq. (2) and its complex conjugate equation only appears via a set of $M$ real collective coordinates $\left\{Y_{1}(t), Y_{2}(t), \ldots Y_{M}(t)\right\}:=\vec{Y}(t)$

$$
u(x, t)=u[x, \vec{Y}(t)], \quad u^{*}(x, t)=u^{*}[x, \vec{Y}(t)] .
$$

Then, we multiply Eq. (2) by $\partial u^{*} / \partial Y_{n}$, and its complex conjugate equation by $\partial u / \partial Y_{n}$, add the resulting equations and integrate over the system, yielding

$$
\sum_{j=1}^{M} I_{Y_{n} Y_{j}} \dot{Y}_{j}=F_{n}(\vec{Y})+R_{n}(\vec{Y}), \quad n=1,2, \ldots M,
$$

with

$$
\begin{aligned}
I_{Y_{n} Y_{j}} & =\int_{-\infty}^{+\infty} d x i\left[\frac{\partial u}{\partial Y_{n}} \frac{\partial u^{*}}{\partial Y_{j}}-\frac{\partial u^{*}}{\partial Y_{n}} \frac{\partial u}{\partial Y_{j}}\right], \\
F_{n}(\vec{Y}) & =-\int_{-\infty}^{+\infty} d x\left[\frac{\delta H_{0}}{\delta u^{*}} \frac{\partial u^{*}}{\partial Y_{n}}+\frac{\delta H_{0}}{\delta u} \frac{\partial u}{\partial Y_{n}}\right] \\
& =-\int_{-\infty}^{+\infty} d x \frac{\partial \mathcal{H}_{0}}{\partial Y_{n}}=-\frac{\partial}{\partial Y_{n}} H_{0}, \\
R_{n}(\vec{Y}) & =-\int_{-\infty}^{+\infty} d x\left(R \frac{\partial u^{*}}{\partial Y_{n}}+R^{*} \frac{\partial u}{\partial Y_{n}}\right),
\end{aligned}
$$

where the overdot denotes the derivative with respect to time. Equations (5)-(8) represent a set of $M$ first-order ODEs for our $M$ CCs. We note that for certain systems it is necessary to assume that the time dependence of the fields can also appear via $\dot{Y}_{1}, \dot{Y}_{2}, \ldots, \dot{Y}_{M}$, which are formally treated in the same way as the CCs. This yields $M$ second-order ODEs for the $M$ CCs. Examples are magnetic vortices [20] and topological solitons in NLKGEs [23,31]; in both cases the width of the excitations depends on the velocity of the excitations.

In order to have an example which can be tested by the perturbation theory based on the IST [14] we now take the one-soliton solution of the unperturbed NLSE and make the following specific ansatz for $u(x, \vec{Y}(t))$ :

$$
u(x, t)=2 i \eta \operatorname{sech}[2 \eta(x-\zeta)] e^{-i[2 \xi(x-\zeta)+\Phi]},
$$

with the 4 CCs soliton position $\zeta(t)$, phase $\Phi(t)$, amplitude $\eta(t)$, and velocity $\xi(t)$. The soliton energy reads

$$
H_{0}=\frac{16}{3} \eta\left(3 \xi^{2}-\eta^{2}\right) \text {. }
$$

Setting $Y_{1}=\zeta, Y_{2}=\Phi, Y_{3}=\eta$ and $Y_{4}=\xi$ in Eqs. (5)-(8), we obtain for $n=1$

$$
8 \dot{\eta} \xi+8 \eta \dot{\xi}=\int_{-\infty}^{+\infty} d x\left(R \frac{\partial u^{*}}{\partial \zeta}+R^{*} \frac{\partial u}{\partial \zeta}\right),
$$

where $2 \eta$ is the soliton mass, and $-4 \xi(t)$ the soliton velocity [30]. Therefore this equation has the form of the Newtonian equation of motion for a point particle with variable mass. The rhs of Eq. (11) represents the time-dependent force obtained by means of the average of the perturbation $R$ over space, where the "weight function" is just the variation of the ansatz with respect to the first $\mathrm{CC}$, the soliton position. In addition to the point-particle representation, the evolution of $\zeta$ and $\Phi$ takes into account other degrees of freedom. Then, in a similar way, i.e., setting $Y_{1}=\zeta, Y_{2}=\Phi, Y_{3}=\eta$ and $Y_{4}=\xi$ in Eqs. (5)-(8), we obtain for $n=2,3,4$

$$
4 \dot{\eta}=-\int_{-\infty}^{+\infty} d x\left(R \frac{\partial u^{*}}{\partial \phi}+R^{*} \frac{\partial u}{\partial \phi}\right),
$$

$$
\begin{aligned}
4(\dot{\Phi}-2 \xi \dot{\zeta}) & \equiv 4(\dot{\phi}+2 \zeta \dot{\zeta}) \\
& =-16\left(\eta^{2}-\xi^{2}\right)+\int_{-\infty}^{+\infty} d x\left(R \frac{\partial u^{*}}{\partial \eta}+R^{*} \frac{\partial u}{\partial \eta}\right),
\end{aligned}
$$

$$
8 \eta \dot{\zeta}=-32 \eta \xi-\int_{-\infty}^{+\infty} d x\left(R \frac{\partial u^{*}}{\partial \xi}+R^{*} \frac{\partial u}{\partial \xi}\right),
$$

respectively, where $\Phi=\phi+2 \zeta \xi$. Hence, the evolution of $\eta$ and $\zeta$ is given directly by Eqs. (12) and (14), respectively. Substituting $\dot{\eta}$ from Eq. (12) in Eq. (11) the equation of motion for $\xi$ is attained. Finally, using the obtained equation for $\dot{\xi}$ in Eq. (13) the time evolution of $\Phi$ is found. Equations (11)-(14) are equivalent to the results of the adiabatic perturbation theory [29]. They are also equivalent to the Eqs. (3.17)-(3.20) of Ref. [14] for $\dot{\eta}, \dot{\xi}, \dot{\zeta}$, and $\dot{\phi}$ obtained by the IST with arbitrary perturbations. However, the GTWM has the following advantages:

(1) It does not require that the IST can be applied to the unperturbed system. In fact, the specific form of $\mathcal{H}_{0}$ in Eq. (3), which is related to the unperturbed NLSE, is not used in the derivation of the evolution Eqs. (5) for the CCs. 
(2) The GTWM works for an arbitrary number of CCs. For example the ansatz of Ref. [37] with 6 CCs can be used. See also the remarks at the end of the conclusions section.

(3) The procedure which yields the evolution Eqs. (5) for the CCs is extremely concise. After having chosen a specific ansatz, the calculation of the integrals Eq. (6) and the forces Eq. (7) is straightforward. The same holds for the perturbation terms Eq. (8) when the perturbation $R$ has been specified.

\section{VARIATIONAL APPROACH AND GTWM}

An advantage of the GTWM is related to the fact that it can be applied in cases where the Lagrangian density $\mathcal{L}\left(u, u^{*}, u_{x}, u_{x}^{*}, u_{t}, u_{t}^{*}\right)$ of the perturbed system is unknown. However, if $\mathcal{L}$ exists and if, in addition, $\mathcal{L}$ and the ansatz Eqs. (4) satisfy certain conditions [see Eqs. (22) and (23) below], we show that the variational approach and the GTWM are equivalent, i.e., both methods yield the same equations of motion for the CCs.

In order to prove this statement, we separate the perturbation $R[u(x, t) ; x, t]$ in two parts

$$
R[u(x, t) ; x, t]=-i \beta u+B[u(x, t) ; x, t],
$$

such that the dissipation in the system appears only in the first term, $-i \beta u$. We assume that the perturbed NLSE Eq. (1) is equivalent to an Euler-Lagrange equation, generalized by a dissipative term on the rhs,

$$
\frac{d}{d t} \frac{\partial \mathcal{L}}{\partial u_{t}^{*}}+\frac{d}{d x} \frac{\partial \mathcal{L}}{\partial u_{x}^{*}}-\frac{\partial \mathcal{L}}{\partial u^{*}}=\frac{\delta \mathcal{F}}{\delta u_{t}^{*}},
$$

with the dissipation function

$$
\mathcal{F}=-i \beta\left(u u_{t}^{*}-u^{*} u_{t}\right),
$$

and similarly for the complex conjugate of Eq. (1). We note that more complicated dissipation terms than the simple term $-i \beta u$ in Eq. (15) can also be treated by generalizing the Euler-Lagrange formalism [17].

Now, inserting the ansatz Eq. (4) in $\mathcal{L}$ and $\mathcal{F}$ [32,33] and integrating, we obtain

$$
\begin{aligned}
& L=\int_{-\infty}^{+\infty} d x \mathcal{L}\left(u, u^{*}, u_{x}, u_{x}^{*}, u_{t}, u_{t}^{*}\right)=L(\vec{Y} ; \dot{\vec{Y}}), \\
& F=\int_{-\infty}^{+\infty} d x \mathcal{F}\left(u, u^{*}, u_{x}, u_{x}^{*}, u_{t}, u_{t}^{*}\right)=F(\vec{Y} ; \dot{\vec{Y}}) .
\end{aligned}
$$

Hence, the $M$ collective coordinates satisfy $M$ generalized Lagrange equations

$$
\frac{d}{d t} \frac{\partial L}{\partial \dot{Y}_{n}}-\frac{\partial L}{\partial Y_{n}}-\frac{\partial F}{\partial \dot{Y}_{n}}=0,
$$

or equivalently

$$
\int_{-\infty}^{+\infty} d x\left[\frac{d}{d t} \frac{\partial \mathcal{L}}{\partial \dot{Y}_{n}}-\frac{\partial \mathcal{L}}{\partial Y_{n}}-\frac{\partial \mathcal{F}}{\partial \dot{Y}_{n}}\right]=0,
$$

where $n=1, \ldots, M$. From now on we denote $Y=Y_{n}$ and assume $\{$ see Eq. (4) in [32]\} that

$$
\begin{gathered}
{\left[\frac{\partial \mathcal{L}}{\partial u_{x}} \frac{\partial u}{\partial Y}\right]_{x \rightarrow+\infty}-\left[\frac{\partial \mathcal{L}}{\partial u_{x}} \frac{\partial u}{\partial Y}\right]_{x \rightarrow-\infty}=0,} \\
{\left[\frac{\partial \mathcal{L}}{\partial u_{x}^{*}} \frac{\partial u^{*}}{\partial Y}\right]_{x \rightarrow+\infty}-\left[\frac{\partial \mathcal{L}}{\partial u_{x}^{*}} \frac{\partial u^{*}}{\partial Y}\right]_{x \rightarrow-\infty}=0 .}
\end{gathered}
$$

As the following relations hold

$$
\begin{gathered}
\frac{d}{d t} \frac{\partial \mathcal{L}}{\partial \dot{Y}}=\frac{d}{d t}\left(\frac{\partial \mathcal{L}}{\partial u_{t}} \frac{\partial u}{\partial Y}\right)+\frac{d}{d t}\left(\frac{\partial \mathcal{L}}{\partial u_{t}^{*}} \frac{\partial u^{*}}{\partial Y}\right), \\
\frac{\partial \mathcal{L}}{\partial Y}=\frac{\partial \mathcal{L}}{\partial u} \frac{\partial u}{\partial Y}+\frac{\partial \mathcal{L}}{\partial u^{*}} \frac{\partial u^{*}}{\partial Y}+\frac{\partial \mathcal{L}}{\partial u_{x}} \frac{\partial u_{x}}{\partial Y} \\
+\frac{\partial \mathcal{L}}{\partial u_{x}^{*}} \frac{\partial u_{x}^{*}}{\partial Y}+\frac{\partial \mathcal{L}}{\partial u_{t}} \frac{\partial u_{t}}{\partial Y}+\frac{\partial \mathcal{L}}{\partial u_{t}^{*}} \frac{\partial u_{t}^{*}}{\partial Y}, \\
\frac{\partial \mathcal{F}}{\partial \dot{Y}}=\frac{\partial \mathcal{F}}{\partial u_{t}} \frac{\partial u}{\partial Y}+\frac{\partial \mathcal{F}}{\partial u_{t}^{*}} \frac{\partial u^{*}}{\partial Y},
\end{gathered}
$$

and taking into account the relations Eqs. (22) and (23), Eq. (21) becomes

$$
\begin{aligned}
\int_{-\infty}^{+\infty} & d x\left\{\frac{d}{d t}\left(\frac{\partial \mathcal{L}}{\partial u_{t}}\right)+\frac{d}{d x}\left(\frac{\partial \mathcal{L}}{\partial u_{x}}\right)-\frac{\partial \mathcal{L}}{\partial u}-\frac{\partial \mathcal{F}}{\partial u_{t}}\right\} \frac{\partial u}{\partial Y} \\
+ & \int_{-\infty}^{+\infty} d x\left\{\frac{d}{d t}\left(\frac{\partial \mathcal{L}}{\partial u_{t}^{*}}\right)+\frac{d}{d x}\left(\frac{\partial \mathcal{L}}{\partial u_{x}^{*}}\right)-\frac{\partial \mathcal{L}}{\partial u^{*}}-\frac{\partial \mathcal{F}}{\partial u_{t}^{*}}\right\} \frac{\partial u^{*}}{\partial Y}=0 .
\end{aligned}
$$

Inserting the Lagrangian density $\mathcal{L}$ and the dissipation function $\mathcal{F}$ in Eq. (27), the first and second curly brackets become the NLS Eq. (1) and its corresponding complex conjugate equation, respectively (up to a constant factor), where $R$ is given by Eq. (15). Then, Eq. (27) represents the integration of the sum of the first NLSE Eq. (1) multiplied by $\partial u / \partial Y$ and the second NLSE, i.e., the complex conjugate of Eq. (1), multiplied by $\partial u^{*} / \partial Y$. This projection procedure is exactly the same as that which has produced the $M$ Eqs. (5) in Sec. II. Thus, the Lagrangian approach is equivalent to the GTWM and vice versa. However, in practice the GTWM requires more work if $M>3$, because $M(M-1) / 2$ integrals $I_{Y_{n} Y_{j}}$ and $M$ integrals $R_{n}(\vec{Y})$ in Eqs. (6) and (8), respectively, must be calculated. In the Lagrangian method one has to perform only $M+3$ integrals to obtain $L$ and $F$. On the other hand, the GTWM is more general since the Lagrangian density of the perturbed system need not be known.

\section{GTWM AND MODIFIED CONSERVED QUANTITIES}

A very peculiar property of GTWMs is related to its relationship with the so-called modified conservation laws (MCL) (the time evolution of the quantities which are conserved for the unperturbed system) [18]. Such an equivalence was already shown for NLKGEs in [23] since usually the 
equations of motion for $\mathrm{M} \mathrm{CCs}$ are related with the first $M$ MCLs.

In this section, we show that in the case of the NLSE the GTWM with the ansatz

$$
u(x, t)=u(x-\zeta, \Phi, \eta, \xi), \quad u^{*}(x, t)=u^{*}(x-\zeta, \Phi, \eta, \xi)
$$

yields at first only 3 MCLs, namely the time variation of the norm, the momentum, and the energy. In Eq. (28) $\zeta$ denotes the soliton position and $\Phi$ represents a phase, (so that $\partial u / \partial \Phi=-i u$ and $\left.\partial u^{*} / \partial \Phi=i u^{*}\right)$. We stress that we need not yet make a specific ansatz for $u$. With $M=4$ and setting $n$ $=1$ in Eqs. (5) $-(8)$ we obtain

$$
\begin{gathered}
\sum_{j=1}^{4} I_{\zeta Y_{j}} \dot{Y}_{j}=\frac{d P}{d t}+\int_{-\infty}^{+\infty} d x \frac{\partial}{\partial x} \frac{i}{2}\left[u_{t} u^{*}-u u_{t}^{*}\right], \\
F_{1}(\zeta, \Phi, \eta, \xi)=\int_{-\infty}^{+\infty} d x \frac{\partial \mathcal{H}_{0}}{\partial x} \\
R_{1}(\zeta, \Phi, \eta, \xi)=\int_{-\infty}^{+\infty} d x\left(R \frac{\partial u^{*}}{\partial x}+R^{*} \frac{\partial u}{\partial x}\right),
\end{gathered}
$$

where $P$ is the momentum

$$
P=\int_{-\infty}^{+\infty} d x \frac{i}{2}\left[u u_{x}^{*}-u^{*} u_{x}\right]
$$

Substituting Eqs. (29)-(31) in Eq. (5) and taking into account that

$$
\int_{-\infty}^{+\infty} d x \frac{\partial}{\partial x}\left\{\frac{i}{2}\left[u_{t} u^{*}-u u_{t}^{*}\right]-\mathcal{H}_{0}\right\}=0,
$$

we obtain

$$
\frac{d P}{d t}=\int_{-\infty}^{+\infty} d x\left(R \frac{\partial u^{*}}{\partial x}+R^{*} \frac{\partial u}{\partial x}\right)
$$

which represents the variation of the momentum with respect to time for the perturbed NLSE Eq. (1) and its complex conjugate equation.

Taking $n=2$ in Eqs. (5)-(8), we obtain

$$
\begin{gathered}
\sum_{j=1}^{4} I_{\Phi Y_{j}} \dot{Y}_{j}=-\frac{d N}{d t}, \quad N=\int_{-\infty}^{+\infty} d x|u|^{2}, \\
F_{2}(\zeta, \Phi, \eta, \xi)=-\frac{\partial H_{0}}{\partial \Phi}=-\frac{\partial}{\partial \Phi} \int_{-\infty}^{+\infty} d x\left(\left|u_{x}\right|^{2}-|u|^{4}\right)=0,
\end{gathered}
$$

$$
\begin{aligned}
R_{2}(\zeta, \Phi, \eta, \xi) & =-\int_{-\infty}^{+\infty} d x\left(R \frac{\partial u^{*}}{\partial \Phi}+R^{*} \frac{\partial u}{\partial \Phi}\right) \\
& =-\int_{-\infty}^{+\infty} d x i\left(R^{*} u-R u^{*}\right) .
\end{aligned}
$$

Hence, substituting Eqs. (35)-(37) in Eq. (5) our second modified conservation law reads

$$
\frac{d N}{d t}=\int_{-\infty}^{+\infty} d x i\left(R^{*} u-R u^{*}\right),
$$

and represents the variation of the norm $N$.

Finally, we insert in Eqs. (5)-(8) $Y_{1}=\zeta, Y_{2}=\Phi, Y_{3}=\eta$, and $Y_{4}=\xi$, with $M=4$. Then, we multiply these equation by $\dot{\zeta}, \dot{\Phi}$, $\dot{\eta}$, and $\dot{\xi}$, respectively; adding them yields

$$
\begin{gathered}
\sum_{n=1}^{4} \sum_{j=1}^{4} I_{Y_{n} Y_{j}} \dot{Y}_{j} \dot{Y}_{n}=0 \\
F_{1} \dot{\zeta}+F_{2} \dot{\Phi}+F_{3} \dot{\eta}+F_{4} \dot{\xi}=-\frac{d H_{0}}{d t}, \\
R_{1} \dot{\zeta}+R_{2} \dot{\Phi}+R_{3} \dot{\eta}+R_{4} \dot{\xi}=-\int_{-\infty}^{+\infty} d x\left(R u_{t}^{*}+R^{*} u_{t}\right),
\end{gathered}
$$

so that the evolution of the energy is given by

$$
\frac{d H_{0}}{d t}=-\int_{-\infty}^{+\infty} d x\left(R u_{t}^{*}+R^{*} u_{t}\right),
$$

which can be written as

$$
\frac{d H_{0}}{d t}=-\sum_{j=1}^{4} \dot{Y}_{j} \int_{-\infty}^{+\infty} d x\left(R \frac{\partial u^{*}}{\partial Y_{j}}+R^{*} \frac{\partial u}{\partial Y_{j}}\right) .
$$

Equation (43) represents the third MCL for NLSE.

We would like to find a fourth MCL, since we are using four CC equations from our GTWM. It is natural to expect that these equations are related not only to the first three conservation laws but also to the fourth one. The infinite sequence of conservation laws of the unperturbed NLSE can be obtained via the following recurrence relation for densities, which was obtained in the framework of the IST [34],

$$
b_{m+1}=u^{*} \frac{d}{d x}\left(\frac{b_{m}}{u^{*}}\right)+\sum_{k+j=m-1} b_{k} b_{j},
$$

with $b_{0}=|u|^{2}, m=0,1, \ldots$ A similar relation, with $b_{m+1}^{*}$, holds for the complex conjugate of Eq. (1) with $R=0$. In this way, for $m=0$ the momentum density $\mathcal{P}=-(i / 2)\left(b_{1}-b_{1}^{*}\right)$ is obtained [34], which is identical with that in Eq. (32). For $m$ $=1$, we obtain the energy density and for $m=2$ a new density,

$$
\rho_{1}=-(i / 2)\left(b_{3}-b_{3}^{*}\right)=\frac{i}{2}\left\{3|u|^{2}\left(u u_{x}^{*}-u_{x} u^{*}\right)+u_{x}^{*} u_{x x}-u_{x} u_{x x}^{*}\right\} .
$$

Using the one-soliton solution for the unperturbed NLSE, i.e., Equation (9) with constant velocity and amplitude and 
the linear functions in time $\zeta=\zeta_{0}-4 \xi t$ and $\Phi=\Phi_{0}$ $-4\left(\xi^{2}+\eta^{2}\right) t$, this yields a new conserved quantity $H_{1}$ $=\int_{-\infty}^{+\infty} d x \rho_{1}(x, t)=32 \eta \xi\left(\xi^{2}-\eta^{2}\right)$. Due to the perturbations $R$ and $R^{*}$ in Eq. (1) and its complex conjugate equation, respectively, the evolution of $H_{1}$ is determined by [18]

$$
\frac{d H_{1}}{d t}=\int_{-\infty}^{+\infty} d x\left[R^{*}\left(6|u|^{2} u_{x}+u_{x x x}\right)+R\left(6|u|^{2} u_{x}^{*}+u_{x x x}^{*}\right)\right],
$$

where $H_{1}$ is the Hamiltonian for the first integrable hierarchy of the NLSE [35]. Comparing the rhs of Eq. (8) with the rhs of Eq. (46) we realize that in the former equation the perturbation $R$ is multiplied by the first derivative of $u^{*}$ with respect to a given CC, whereas in the latter one the third spatial derivative of $u^{*}$ appears. So, the MCL Eq. (46) is not equivalent to any equation or combination of equations of the system Eqs. (5)-(8) obtained by means of GTWM.

However, we can achieve our goal by considering the evolution of the first moment of the norm [19],

$$
\frac{d N_{1}}{d t}=2 P+\int_{-\infty}^{+\infty} d x x\left(R^{*} u-R u^{*}\right), \quad N_{1}=\int_{-\infty}^{+\infty} d x x|u|^{2} .
$$

We now show that Eq. (47) together with the first three MCLs yields the four CC Eqs. (11)-(14). Here we use the specific ansatz Eq. (9) for which the norm

$$
N=4 \eta,
$$

the first moment of the norm,

$$
N_{1}=4 \eta \zeta,
$$

the momentum

$$
P=-8 \eta \xi,
$$

and the energy is given by Eq. (10). Substituting Eqs. (48)-(50) and (10) in the Eqs. (38), (34), (47), and (43), respectively, we obtain

$$
\begin{gathered}
4 \dot{\eta}=\int_{-\infty}^{+\infty} d x i\left(R^{*} u-R u^{*}\right) \\
-8 \eta \dot{\xi}-8 \xi \dot{\eta}=\int_{-\infty}^{+\infty} d x\left(R \frac{\partial u^{*}}{\partial x}+R^{*} \frac{\partial u}{\partial x}\right) \\
4 \eta \dot{\zeta}+4 \zeta \dot{\eta}=-16 \eta \dot{\xi}+i \int_{-\infty}^{+\infty} d x x\left(R^{*} u-R u^{*}\right) \\
-16\left(\eta^{2}-\xi^{2}\right) \dot{\eta}+32 \eta \dot{\xi} \dot{\xi}=-\sum_{j=1}^{4} \dot{Y}_{j} \int_{-\infty}^{+\infty} d x\left(R \frac{\partial u^{*}}{\partial Y_{j}}+R^{*} \frac{\partial u}{\partial Y_{j}}\right) .
\end{gathered}
$$

From Eq. (51) and the identity $\partial u / \partial \xi=-2 i(x-\zeta) u$, Eq. (53) becomes

$$
8 \eta \dot{\zeta}=-32 \eta \xi-\int_{-\infty}^{+\infty} d x\left(R \frac{\partial u^{*}}{\partial \xi}+R^{*} \frac{\partial u}{\partial \xi}\right)
$$

which provides the evolution equation for $\dot{\zeta}$. Taking into account Eqs. (51), (52), and (55), Eq. (54) becomes

$$
\dot{\eta}\left\{4(\dot{\Phi}-2 \dot{\xi} \dot{\zeta})-16\left(\xi^{2}-\eta^{2}\right)-\int_{-\infty}^{+\infty} d x\left(R \frac{\partial u^{*}}{\partial \eta}+R^{*} \frac{\partial u}{\partial \eta}\right)\right\}=0,
$$

yielding the evolution equation for $\Phi$ since $\eta$ evolves in time. From Eqs. (51) and (52) we obtain the equation of motion for $\eta$ and $\xi$. From Eqs. (55) and (56), the equations for $\zeta$ and $\Phi$ can be obtained. In fact, these four equations agree with Eqs. (11)-(14) obtained via GTWM.

\section{CONCLUSIONS}

We have developed the GTWM for the perturbed NLS Eqs. (1) and the complex conjugate of Eq. (1) (this is different from earlier applications of the method which started from the Hamilton equations for magnetic vortices and for solitons in NLKGEs). The GTWM is based on a projection technique: The NLSEs Eq. (2) and the complex conjugate of Eq. (2) are multiplied by derivatives of $u^{*}$ and $u$ with respect to one of the CCs, respectively. Then the resulting equations are added and integrated over the system. This yields the evolution equations for an arbitrary number of CCs, Eqs. (5)-(8), without using a specific ansatz. This method is more general than the variational approach because it is not necessary to know the Lagrangian density $\mathcal{L}$ of the perturbed system. E.g., see Eq. (1) of [36], where the Lagrangian density does not exist, but the Hamiltonian $H_{0}$ of the unperturbed system is known. In cases where $\mathcal{L}$ exists and conditions Eqs. (22) and (23) are satisfied, we have shown that the GTWM and the variational formalism are equivalent, i.e., from both theories the same evolution equations for the $\mathrm{CCs}$ are obtained.

Using 4 collective coordinates with the ansatz Eq. (9), namely, the position $\zeta$, velocity $\xi$, amplitude $\eta$ and phase $\Phi$ of the soliton, we have explicitly shown that the GTWM is equivalent to the results obtained from the IST, from the adiabatic perturbation theory and from the time variation of the norm, the first moment of the norm, the momentum, and the energy of the system. However, we stress that the GTWM is very concise, and more general than the IST and the time-variation of the conserved quantities since it can be applied when we have an ansatz with more than 4 CCs (see the ansätze with 5 CCs in Refs. [36,38] for soliton propagation in optical fibers and with 6 CCs used in [37]). Moreover, the perturbations considered in Refs. [36,38] include several dissipative terms which present no additional difficulty for the GTWM, in contrast to the variational method. Work along this line is in progress.

\section{ACKNOWLEDGMENTS}

We thank Yuri Gaididei (Kiev) and Edward Arevalo (Dresden) for very useful discussions on this work. N.R.Q. 
acknowledges financial support by the Ministerio de Educación y Ciencia (MEC, Spain) through Grant No. FIS200802380/FIS, and by the Junta de Andalucía under Project Nos. FQM207, FQM-00481, P06-FQM-01735, and P09-FQM4643. F.G.M. acknowledges the hospitality of the University of Seville and of the Theoretical Division and Center for Nonlinear Studies at Los Alamos Laboratory. Work at Los Alamos was supported by U.S. DOE. F.G.M. acknowledges financial support by IMUS and by the Plan Propio of the University of Seville.
[1] A. R. Bishop, J. A. Krumhansl, and S. E. Trullinger, Physica D 1, 1 (1980).

[2] A. C. Scott, Nonlinear Science (Oxford University, Oxford, 1999).

[3] D. W. McLaughlin and A. C. Scott, Phys. Rev. A 18, 1652 (1978).

[4] A. Sánchez and A. R. Bishop, SIAM Rev. 40, 579 (1998).

[5] M. J. Rice and E. J. Mele, Solid State Commun. 35, 487 (1980).

[6] M. Salerno and A. C. Scott, Phys. Rev. B 26, 2474 (1982).

[7] V. Stehr, P. Müller, F. G. Mertens, and A. R. Bishop, Phys. Rev. E 79, 036601 (2009).

[8] M. B. Fogel, S. E. Trullinger, A. R. Bishop, and J. A. Krumhansl, Phys. Rev. B 15, 1578 (1977).

[9] A. Bondeson, M. Lisak, and D. Anderson, Phys. Scr. 20, 479 (1979).

[10] D. J. Kaup and A. C. Newell, Proc. R. Soc. London, Ser. A 361, 413 (1978).

[11] D. J. Kaup, Phys. Rev. A 42, 5689 (1990).

[12] J. Yan, Y. Tang, G. Zhou, and Z. Chen, Phys. Rev. E 58, 1064 (1998).

[13] V. I. Karpman and E. M. Maslov, Sov. Phys. JETP 46, 281 (1977).

[14] Y. Kivshar and B. Malomed, Rev. Mod. Phys. 61, 763 (1989).

[15] B. Malomed, in Progress in Optics, edited by E. Wolf (NorthHolland, Amsterdam, 2002), Vol. 43, p. 71.

[16] A. Hasegawa, Chaos 10, 475 (2000).

[17] S. Chávez Cerda, S. B. Cavalcanti, and J. M. Hickmann, Eur. Phys. J. D 1, 313 (1998).

[18] V. I. Karpman, Phys. Scr. 20, 462 (1979).

[19] W. L. Kath, Methods Appl. Anal. 4, 141 (1997).

[20] F. G. Mertens, H. J. Schnitzer, and A. R. Bishop, Phys. Rev. B 56, 2510 (1997).

[21] T. Kamppeter, F. G. Mertens, A. Sánchez, F. DomínguezAdame, A. R. Bishop, and N. Gronbeck-Jensen, Eur. Phys. J.
B 7, 607 (1999).

[22] T. Kamppeter, F. G. Mertens, E. Moro, A. Sánchez, and A. R. Bishop, Phys. Rev. B 59, 11349 (1999).

[23] N. R. Quintero, A. Sánchez, and F. G. Mertens, Phys. Rev. E 62, 5695 (2000).

[24] N. R. Quintero, A. Sánchez, and F. G. Mertens, Phys. Rev. Lett. 84, 871 (2000).

[25] N. R. Quintero, A. Sánchez, and F. G. Mertens, Phys. Rev. E 62, R60 (2000).

[26] L. Morales-Molina, N. R. Quintero, F. G. Mertens, and A. Sánchez, Phys. Rev. Lett. 91, 234102 (2003).

[27] L. Morales-Molina, N. R. Quintero, A. Sánchez, and F. G. Mertens, Chaos 16, 013117 (2006).

[28] A. V. Ustinov, C. Coqui, A. Kemp, Y. Zolotaryuk, and M. Salerno, Phys. Rev. Lett. 93, 087001 (2004).

[29] G. P. Agrawal, Nonlinear Fiber Optics (Academic, San Diego, 2001).

[30] L. D. Faddeev and L. Takhtajan, Hamiltonian Methods in the Theory of Solitons (Classics in Mathematics) (Springer-Verlag, Berlin, 2007).

[31] L. Morales-Molina, F. G. Mertens, and A. Sánchez, Phys. Rev. E 72, 016612 (2005).

[32] O. Legrand, Phys. Rev. A 36, 5068 (1987).

[33] N. R. Quintero and E. Zamora-Sillero, Physica D 197, 63 (2004).

[34] S. P. Novikov, S. V. Manakov, L. P. Pitaevskii, and V. E. Zakharov, Theory of Solitons: The Inverse Scattering Method (Springer-Verlag, Berlin, 1984).

[35] A. Hasegawa and Y. Kodama, Solitons in Optical Communications (Oxford, New York, 1995), pp. 70-72.

[36] J. Santhanam and Govind P. Agrawal, Opt. Commun. 222, 413 (2003).

[37] A. V. Maimistov, Sov. Phys. JETP 77, 727 (1993).

[38] Z. Chen, A. J. Taylor, and A. Efimov, J. Opt. Soc. Am. B 27, 1022 (2010). 